\title{
QUAN NIỆM HỌC TẬP NGOẠI NGŨ̃ THỨ HAI - TIẾNG TRUNG QUỐC CỦA SINH VIÊN NGÀNH NGÔN NGŨ ANH, TRƯờnG ĐẠI HỌC NGÂN HÀNG TP. HỒ CHÍ MINH
}

\author{
Lưu Hớn Vũ \\ Khoa Ngoại ngũu, Truờng Đại học Ngân hàng TP. Hồ Chí Minh \\ 36 Tôn Thất Đạm, Quận 1, TP. Hồ Chí Minh, Việt Nam \\ Nhận bài ngày 2 tháng 2 năm 2020 \\ Chỉnh sửa ngày 12 tháng 5 năm 2020; Chấp nhận đăng ngày 25 tháng 9 năm 2020
}

Tóm tắt: Bài viết khảo sát quan niệm học tập ngoại ngữ thứ hai - tiếng Trung Quốc của sinh viên ngành Ngôn ngữ Anh, Trường Đại học Ngân hàng TP. Hồ Chí Minh. Trên cơ sở lí thuyết về quan niệm học tập của Horwitz (1985), chúng tôi tiến hành khảo sát bằng bảng hỏi với 177 sinh viên. Kết quả cho thấy quan niệm học tập của sinh viên như sau: thứ nhất, tiếng Trung Quốc tương đối dễ học; thứ hai, trẻ em có năng lực học tập ngoại ngữ tốt hơn người lớn; thứ ba, chú trọng ngữ âm, từ vựng và văn hoá, không chú trọng ngữ pháp; thứ tư, học tiếng Trung Quốc có ích cho bản thân. Sinh viên nữ chú trọng về ngữ âm hơn sinh viên nam. Sinh viên năm thứ hai chú trọng về ngữ âm hơn sinh viên năm thứ ba, song lại không chú trọng về ngữ pháp như sinh viên năm thứ ba. Khác với sinh viên miền trung, sinh viên miền bắc và miền nam cho rằng nên đến Trung Quốc học tiếng Trung Quốc. Quan niệm "tiếng Trung Quốc dễ học", thái độ tự tin và chủ động sử dụng tiếng Trung Quốc có ảnh hưởng tích cực đến kết quả học tập của sinh viên.

Tù khoá: quan niệm học tập; tiếng Trung Quốc; ngoại ngữ thứ hai

\section{1. Đặt vấn đề}

Trong những năm gần đây, sự khác biệt cá thể của người học đã trở thành vấn đề rất được quan tâm trong lĩnh vực thụ đắc ngôn ngữ thứ hai. Trong đó, quan niệm học tập (learning beliefs) là một nhân tố khác biệt cá thể quan trọng. Quan niệm học tập là giả thiết thông thường của người học về bản thân, về nhân tố ảnh hưởng học tập và về bản chất của việc dạy và học (Victori \& Lockheart, 1995), là những thông tin về bản chất học tập, quá trình học tập và tình hình bản thân người học mà người học có được thông qua việc người học tự trải nghiệm hoặc do ảnh hưởng của người khác, và là hệ thống quan điểm làm thế nào để nắm

\footnotetext{
ĐT: 84-825159698
}

Email: luuhonvu@gmail.com vững kiến thức ngôn ngữ, kĩ năng ngôn ngữ và năng lực giao tiếp (Wenden, 1991). Việc hình thành quan niệm học tập chịu ảnh hưởng của nhiều nhân tố như nhân tố xã hội, nhân tố văn hoá, nhân tố tri nhận, nhân tố tình cảm, nhân tố cá thể (Bernat, 2006). Quan niệm học tập sẽ thay đổi cùng với sự thay đổi của môi trường học tập (Amuzie \& Winke, 2009).

Hiện nay, đã có một số công trình nghiên cứu về quan niệm học tập tiếng Trung Quốc của sinh viên quốc tế tại Trung Quốc như các nghiên cứu của Ding An-qi (丁安琪) (2010), Lin Lun-lun (林伦伦) và Ren Meng-ya (任梦 雅) (2010), Ding An-qi (丁安琪) và Wu Sina (吴思娜) (2011)... Song, trong các tài liệu mà chúng tôi thu thập được, thành quả nghiên cứu về quan niệm học tập tiếng Trung Quốc của sinh viên Việt Nam nói chung, sinh viên 
học ngoại ngữ thứ hai - tiếng Trung Quốc ${ }^{1}$ nói riêng, vẫn còn rất hạn chế.

Trong phạm vi bài nghiên cứu này, chúng tôi mong muốn tìm câu trả lời cho ba vấn đề sau: Thứ nhất, quan niệm học tập ngoại ngữ thứ hai - tiếng Trung Quốc của sinh viên ngành Ngôn ngữ Anh, Trường Đại học Ngân hàng TP. Hồ Chí Minh như thế nào? Thứ hai, các nhân tố cá thể (giới tính, thời gian học, vùng miền) có ảnh hưởng đến quan niệm học tập ngoại ngữ thứ hai - tiếng Trung Quốc của sinh viên ngành Ngôn ngữ Anh, Trường Đại học Ngân hàng TP. Hồ Chí Minh không? Thứ ba, mối quan hệ giữa kết quả học tập với quan niệm học tập ngoại ngữ thứ hai - tiếng Trung Quốc của sinh viên như thế nào?

\section{Lịch sử nghiên cứu vấn đề}

Từ những năm 70 của thế kỉ XX, quan niệm học tập ngôn ngũ̃ đã trở thành vấn đề được các nhà ngôn ngữ học, giáo dục học quan tâm nghiên cứu. Các nhà nghiên cứu khác nhau đã có những cách phân loại quan niệm học tập ngôn ngữ khác nhau. Horwitz (1985) trong Bảng điều tra quan niệm học tập ngôn ngữ (Belief About Language Learning Inventory, BALLI) đã chia quan niệm học tập ngôn ngữ thành năm phương diện: năng lực học tập ngoại ngữ, độ khó của việc học ngoại ngữ, tính chất của việc học ngoại ngữ, chiến lược học tập - giao tiếp và động cơ học tập. Wenden (1987) cho rằng, quan niệm học tập ngôn ngữ có thể phân thành năm lĩnh vực: ngôn ngữ, trình độ người học, kết quả nỗ lực học tập của người học, tác dụng của người học trong quá trình học tập ngôn ngữ, con

\footnotetext{
1 Ngoại ngữ thứ hai - tiếng Trung Quốc là ngoại ngữ tự chọn dành cho sinh viên thuộc nhóm ngành Ngôn ngữ, văn học và văn hoá nước ngoài (Mã nhóm 72202), nhưng không học ngành Ngôn ngũ̃ Trung Quốc (Mã ngành 7220204).
}

đường tốt nhất để hoàn thành nhiệm vụ học tập ngôn ngữ. Richard và Lockhart (1994) lại chia quan niệm học tập ngôn ngữ thành tám loại: quan niệm về tính chất ngôn ngữ, quan niệm về người bản ngữ, quan niệm về bốn loại kĩ năng, quan niệm về giảng dạy, quan niệm về học tập, quan niệm về tính thích hợp của hành vi trên lớp, quan niệm về tính tự thân và quan niệm về mục tiêu học tập. Trong đó, cách phân loại của Horwitz và Bảng điều tra BALLI của ông được đánh giá rất cao, có tầm ảnh hưởng khá lớn, được sử dụng rộng rãi trong nhiều nghiên cứu khác nhau (Peacock, 2001; Jee, 2014).

Không chỉ làm rõ các đặc điểm về quan niệm học tập ngôn ngữ của người học, các nhà nghiên cứu còn tìm hiểu mối quan hệ giữa các nhân tố khác với quan niệm học tập ngôn ngữ của người học. Bacon và Finnemann (1990), Bernat và Lloyd (2007) đã tiến hành nghiên cứu ảnh hưởng của nhân tố giới tính, thời gian học đối với quan niệm học tập ngôn ngữ của người học. Mori (1999) đã nghiên cứu mối tương quan giữa quan niệm học tập và kết quả học tập của người học. Tanaka và Ellis (2003) đã nghiên cứu ảnh hưởng của môi trường ngôn ngữ đối với quan niệm học tập ngôn ngữ của người học.

Nghiên cứu về quan niệm học tập tiếng Trung Quốc chỉ mới bắt đầu từ những năm đầu của thế kỉ XXI, số lượng công trình nghiên cứu vẫn còn rất hạn chế. Cao Xian-wen (曹贤 文) và Wu Huai-nan (吴淮南) (2002) nghiên cứu về quan niệm học tập tiếng Trung Quốc của sinh viên quốc tế tại Trung Quốc. Sau công trình này, các nghiên cứu về quan niệm học tập tiếng Trung Quốc đều hướng đến đối tượng người học cụ thể, như sinh viên Hàn Quốc (Wu Yan (吴艳) và Sun Li-ming (孙莉 明), 2010; Ding An-qi (丁安琪), 2010), sinh viên Nhật Bản (Mii Akiko (三井明子) và Shao Ming-ming (邵明明), 2019), sinh viên 
Malaysia (Ding An-qi (丁安琪) và Wu Si-na ( 吴思娜), 2011), sinh viên châu Phi (Lin Lunlun (林伦伦) và Ren Meng-ya (任梦雅), 2010), sinh viên khu vực Trung Á (Zhang Hui (张慧), 2011)... Kết quả của các nghiên cứu này cho thấy, sinh viên đến từ các quốc gia khác nhau có những quan niệm học tập tiếng Trung Quốc khác nhau.

\section{Phương pháp nghiên cứu}

\subsection{Khách thể nghiên cứu}

Tham gia điều tra là 177 sinh viên ngành Ngôn ngữ Anh của Trường Đại học Ngân hàng TP. Hồ Chí Minh đang học ngoại ngữ thứ hai - tiếng Trung Quốc. Trong đó, có 21 sinh viên nam (chiếm tỉ lệ $11.9 \%$ ) và 156 sinh viên nữ (chiếm tỉ lệ $88.1 \%$ ); có 86 sinh viên năm thứ hai (chiếm tỉ lệ 48.6\%) và 91 sinh viên năm thứ ba (chiếm tî̉ lệ 51.4\%); có 14 sinh viên đến từ các tỉnh, thành miền bắc (chiếm tỉ lệ $7.9 \%), 105$ sinh viên đến từ các tỉnh, thành miền trung (chiếm tỉ lệ $59.3 \%$ ) và 58 sinh viên đến từ các tỉnh, thành miền nam (chiếm tỉ lệ $32.8 \%)$. Sinh viên có độ tuổi thấp nhất là 19 tuổi, độ tuổi cao nhất là 23 tuổi, độ tuổi trung bình là 19.68 tuổi.

\subsection{Công cụ thu thập dĩu liệu}

Chúng tôi sử dụng công cụ Bảng điều tra BALLI của Horwitz (1985) để khảo sát quan niệm học tập ngoại ngữ thứ hai - tiếng Trung Quốc của sinh viên ngành Ngôn ngữ Anh, Trường Đại học Ngân hàng TP. Hồ Chí Minh. Phiếu điều tra có tổng cộng 34 câu hỏi, sử dụng thang đo năm bậc của Likert từ "hoàn toàn không đồng ý" đến "hoàn toàn đồng ý". Các câu hỏi xoay quanh năm phương diện: năng lực học tập ngoại ngữ (bao gồm các câu Q1, Q2, Q10, Q15, Q22, Q29, Q32, Q33 và Q34), độ khó của việc học ngoại ngữ (bao gồm các câu Q3, Q4, Q6, Q24 và Q28), tính chất của việc học ngoại ngữ (bao gồm các câu
Q5, Q8, Q11, Q16, Q20, Q25 và Q26), chiến lược học tập - giao tiếp (bao gồm các câu Q7, Q9, Q12, Q13, Q17, Q18, Q19 và Q21), động cơ học tập (bao gồm các câu Q23, Q27, Q30 và Q31).

\subsection{Quá trình điều tra}

Chúng tôi tiến hành điều tra bằng bản giấy vào tháng 12 năm 2019 tại Khoa Ngoại ngữ, Trường Đại học Ngân hàng TP. Hồ Chí Minh. Trước khi phát phiếu điều tra, chúng tôi thông báo với sinh viên kết quả điều tra này không ảnh hưởng đến kết quả học tập của sinh viên, hi vọng sinh viên căn cứ vào tình hình thực tế của bản thân trả lời đầy đủ tất cả các câu hỏi có trong phiếu.

Chúng tôi phát ra 177 phiếu, thu vào 177 phiếu, tỉ lệ thu vào $100 \%$. Tất cả các phiếu thu vào đều là phiếu hợp lệ, sinh viên trả lời đầy đủ tất cả các câu hỏi có trong phiếu, đạt tỉ lệ $100 \%$.

\subsection{Công cụ phân tích số liệu}

Chúng tôi sử dụng phần mềm SPSS (phiên bản 25.0) để thống kê, phân tích số liệu mà chúng tôi thu thập được. Trong bài viết này, chúng tôi sử dụng SPSS trong các thống kê mô tả, kiểm định giả thuyết về trị trung bình của hai tổng thể - trường hợp mẫu độc lập (Independent - samples T-test), phân tích phương sai một yếu tố (oneway ANOVA) và phân tích tương quan Pearson.

\section{Kết quả và thảo luận}

\section{1. Đặc điểm chung về quan niệm học tập}

\subsubsection{Năng lục học tập ngoại ngũu}

Trong BALLI có 9 câu hỏi về phương diện năng lực học tập ngoại ngữ. Tỉ lệ phần trăm của các lựa chọn, Mean và SD của 9 câu hỏi về phương diện này như sau (xem bảng 1$)$ : 
Bảng 1. Kết quả điều tra về năng lực học tập ngoại ngữ

\begin{tabular}{|c|c|c|c|c|c|c|c|}
\hline \multirow{2}{*}{ Mã câu hỏi } & \multicolumn{5}{|c|}{ Tỉ lệ phần trăm } & \multirow{2}{*}{ Mean } & \multirow{2}{*}{ SD } \\
\hline & 1 & 2 & 3 & 4 & 5 & & \\
\hline Q1 & 0 & 3.4 & 2.8 & 25.4 & 68.4 & 4.59 & 0.711 \\
\hline Q2 & 1.7 & 1.1 & 7.9 & 44.1 & 45.2 & 4.30 & 0.802 \\
\hline Q10 & 7.9 & 17.5 & 29.4 & 31.1 & 14.1 & 3.26 & 1.143 \\
\hline Q15 & 15.8 & 21.5 & 46.9 & 14.7 & 1.1 & 2.64 & 0.956 \\
\hline Q22 & 40.7 & 12.4 & 39.5 & 5.1 & 2.3 & 2.16 & 1.091 \\
\hline Q29 & 40.1 & 28.2 & 20.3 & 9.0 & 2.3 & 2.05 & 1.083 \\
\hline Q32 & 3.4 & 9.6 & 26.6 & 41.2 & 19.2 & 3.63 & 1.009 \\
\hline Q33 & 3.4 & 13.0 & 63.8 & 16.4 & 3.4 & 3.03 & 0.753 \\
\hline Q34 & 1.1 & 7.9 & 9.0 & 33.3 & 48.6 & 4.20 & 0.979 \\
\hline
\end{tabular}

Bảng 1 cho thấy sinh viên tán thành các quan niệm "Trẻ em học ngoại ngữ dễ hơn người lớn” (Q1, Mean = 4.59), "Có một số người có khả năng bẩm sinh đặc biệt trong việc học ngoại ngữ" (Q2, Mean = 4.30), "Ai cũng có thể học tốt một ngoại ngữ” (Q34, Mean $=4.2$ ), không tán thành các quan niệm "Tôi có khả năng đặc biệt trong việc học ngoại ngữ" (Q15, Mean = 2.64), "Nữ giới học ngoại ngữ giỏi hơn nam giới” (Q22, Mean = 2.16), "Người giỏi về toán và khoa học tự nhiên, không giỏi trong việc học ngoại ngữ" (Q29, Mean = 2.05). Qua đó có thể thấy đại đa số sinh viên cho rằng tồn tại cái gọi là năng lực học tập ngoại ngữ, song đại đa số sinh viên đều cho rằng bản thân mình không có khả năng đặc biệt trong việc học ngoại ngữ. Kết quả này giống với kết quả nghiên cứu của Ding Anqi (丁安琪) (2010) về trường hợp sinh viên Hàn Quốc học tiếng Trung Quốc, song không giống với kết quả của Ding An-qi (丁安琪) và Wu Si-na (吴思娜) (2011) về trường hợp sinh viên Malaysia học tiếng Trung Quốc.

Đại đa số sinh viên hoàn toàn đồng ý hoặc đồng ý quan niệm "Trẻ em học ngoại ngữ dễ hơn người lớn" (Q1, chiếm tỉ lệ 93.8\%). Qua đó cho thấy sinh viên cho rằng tuổi tác có ảnh hưởng đến việc học ngoại ngữ. Kết quả này giống với kết quả nghiên cứu của Ding An-qi (丁安琪) (2010), Ding An-qi (丁安琪) và Wu
Si-na (吴思娜) (2011) về trường hợp sinh viên Hàn Quốc, Malaysia học tiếng Trung Quốc. Song, quan niệm này không hữu ích cho việc học ngoại ngữ của sinh viên. Sinh viên có thể sẽ cho rằng việc gặp khó khăn trong học tập ngoại ngữ và việc có kết quả học tập ngoại ngữ không tốt là do bản thân đã qua độ tuổi tốt nhất để học ngoại ngữ (Ding An-qi (丁安琪), 2010).

Tuy đại đa số sinh viên hoàn toàn đồng ý hoặc đồng ý quan niệm "Có một số người có khả năng bẩm sinh đặc biệt trong việc học ngoại ngữ" (Q2, chiếm tỉ lệ 89.3\%), song họ cũng hoàn toàn đồng ý hoặc đồng ý quan niệm “Ai cũng có thể học tốt một ngoại ngữ" (Q34, chiếm tỉ lệ $81.9 \%$ ). Điều này có thể là do quan niệm "cần cù bù thông minh" của người Việt Nam, thông qua sự chăm chỉ, nỗ lực sẽ giúp bù trừ những thiếu sót về mặt năng lực, từ đó hoàn toàn có thể đạt được những mục tiêu mong muốn.

Đại đa số sinh viên hoàn toàn không đồng ý hoặc không đồng ý quan niệm "Nữ giới học ngoại ngữ giỏi hơn nam giới” (Q22, chiếm tỉ 1ệ $53.1 \%$ ) và "Người giỏi về toán và khoa học tự nhiên, không giỏi trong việc học ngoại ngữ" (Q29, chiếm tỉ lệ 68.3\%). Điều này cho thấy họ không cho rằng giới tính có ảnh hưởng đến việc học ngoại ngữ, cũng như không cho rằng thiên phú về khoa học tự nhiên có ảnh hưởng đến việc học ngoại ngữ. 
4.1.2. Độ khó của việc học ngoại ngũu

Trong BALLI có 6 câu hỏi về phương diện độ khó của việc học ngoại ngữ. Tỉ lệ phần trăm của các lựa chọn, Mean và SD của 6 câu hỏi này như sau (xem bảng 2 ):

Bảng 2. Kết quả điều tra về độ khó của việc ngoại ngữ

\begin{tabular}{|c|c|c|c|c|c|c|c|}
\hline \multirow{2}{*}{ Mã câu hỏi } & \multicolumn{5}{|c|}{ Tỉ lệ phần trăm } & \multirow[b]{2}{*}{ Mean } & \multirow[b]{2}{*}{ SD } \\
\hline & 1 & 2 & 3 & 4 & 5 & & \\
\hline Q3 & 0.6 & 6.2 & 2.8 & 33.3 & 57.1 & 4.40 & 0.861 \\
\hline Q4 & 4.0 & 22.0 & 18.6 & 46.9 & 8.5 & 3.34 & 1.038 \\
\hline Q6 & 1.1 & 5.6 & 18.6 & 48.6 & 26.0 & 3.93 & 0.879 \\
\hline $\mathrm{Q} 14^{1}$ & 9.6 & 41.2 & 29.9 & 9.0 & 10.2 & / & l \\
\hline Q24 & 5.1 & 17.5 & 27.7 & 29.4 & 20.3 & 3.42 & 1.146 \\
\hline Q28 & 28.8 & 39.0 & 12.4 & 14.7 & 5.1 & 2.28 & 1.177 \\
\hline
\end{tabular}

Bảng 2 cho thấy đại đa số sinh viên cho rằng có ngoại ngữ dễ học, có ngoại ngữ khó học $(\mathrm{Q} 3$, Mean $=4.40)$ và tiếng Trung Quốc là ngoại ngữ tương đối dễ học $(\mathrm{Q} 4$, Mean = $3.34)$, tin rằng mình có thể học tốt tiếng Trung Quốc $(\mathrm{Q} 6$, Mean = 3.93). Muốn sử dụng thành thạo tiếng Trung Quốc, nếu mỗi ngày chỉ học một giờ tiếng Trung Quốc, có $41.2 \%$ sinh viên cho rằng phải học $1-2$ năm, $29.9 \%$ sinh viên cho rằng phải học $3-5$ năm, $9.6 \%$ sinh viên cho rằng không cần đến 1 năm, $9 \%$ sinh viên cho rằng cần $5-10$ năm, $10.2 \%$ sinh viên cho rằng đó là điều không thể (Q14). Kết quả này không giống với kết quả nghiên cứu của Ding An-qi (丁安琪) (2010), Ding An-qi (丁安琪) và Wu Si-na (吴思娜) (2011) về trường hợp sinh viên Hàn Quốc, sinh viên Malaysia học tiếng Trung Quốc. Sinh viên Hàn Quốc và sinh viên Malaysia đều cho rằng tiếng Trung Quốc là ngôn ngữ tương đối khó học, đại đa số đều cho rằng cần 3-5 năm mới có thể sử dụng thành thạo tiếng Trung Quốc. Sự khác biệt này có thể là vì loại hình ngôn ngữ của tiếng Việt và tiếng Trung Quốc giống nhau, đều là loại hình đơn lập, trong khi đó loại hình ngôn ngữ của tiếng Hàn Quốc và tiếng Malaysia đều là loại hình chắp dính, khác với loại hình ngôn ngữ của

Đây là câu hỏi lựa chọn 5 phương án cho sẵn, không phải câu hỏi lựa chọn mức độ đồng ý, vì vậy chúng tôi chỉ tính tỉ lệ phần trăm của các lựa chọn, không tính Mean và SD. tiếng Trung Quốc. "Học một ngoại ngữ có đặc điểm loại hình gần với tiếng mẹ đẻ sẽ dễ hơn là học một ngoại ngữ khác xa về loại hình" (Bùi Mạnh Hùng, 2008).

Đa số sinh viên đều cho rằng nói một ngoại ngữ dễ hơn nghe, đọc, viết ngoại ngữ đó (Q24, Mean = 3.42). Trong bốn kĩ năng ngôn ngữ nghe, nói, đọc, viết, khá ít sinh viên cho rằng đọc và viết tiếng Trung Quốc dễ hơn nghe và nói tiếng Trung Quốc (Q28, Mean = 2.28). Kết quả này giống với kết quả khảo sát của Gao Yan-de (高彦德), Li Guo-qiang ( 李国强) và Guo Xu (郭旭) (1993) về trường hợp sinh viên quốc tế tại Trung Quốc. Ngoài ra, kết quả khảo sát của Gao Yan-de (高彦 德), Li Guo-qiang (李国强) và Guo Xu (郭旭) (1993) còn cho thấy, chữ Hán - loại hình văn tự biểu ý "khó nhớ và khó viết" là một trong những yếu tố gây trở ngại trong việc thực hiện kĩ năng đọc, viết tiếng Trung Quốc của sinh viên. Đây cũng có thể là nguyên nhân làm cho sinh viên ngoại ngữ thứ hai - tiếng Trung Quốc cảm thấy đọc, viết tiếng Trung Quốc khó hơn nghe, nói tiếng Trung Quốc.

\subsubsection{Tính chất của việc học ngoại ngũ}

Trong BALLI có 7 câu hỏi về phương diện tính chất của việc học ngoại ngữ. Tỉ lệ phần trăm của các lựa chọn, Mean và $\mathrm{SD}$ của 7 câu hỏi này như sau (xem bảng 3 ): 
Bảng 3. Kết quả điều tra về tính chất của việc ngoại ngữ

\begin{tabular}{|c|c|c|c|c|c|c|c|}
\hline \multirow{2}{*}{ Mã câu hỏi } & \multicolumn{5}{|c|}{ Tỉ lệ phần trăm } & \multirow{2}{*}{ Mean } & \multirow{2}{*}{ SD } \\
\hline & 1 & 2 & 3 & 4 & 5 & & \\
\hline Q5 & 6.2 & 53.1 & 8.5 & 23.7 & 8.5 & 2.75 & 1.141 \\
\hline Q8 & 0.6 & 3.4 & 9.0 & 43.5 & 43.5 & 4.26 & 0.805 \\
\hline Q11 & 14.7 & 31.1 & 9.6 & 29.4 & 15.2 & 2.99 & 1.346 \\
\hline Q16 & 0.6 & 3.4 & 1.1 & 28.8 & 66.1 & 4.56 & 0.737 \\
\hline Q20 & 15.3 & 30.5 & 28.2 & 19.8 & 6.2 & 2.71 & 1.134 \\
\hline Q25 & 3.4 & 9.0 & 35.6 & 32.2 & 19.8 & 3.56 & 1.016 \\
\hline Q26 & 13.6 & 41.8 & 25.4 & 16.4 & 2.8 & 2.53 & 1.012 \\
\hline
\end{tabular}

Bảng 3 cho thấy đại đa số sinh viên không cho rằng tiếng Việt và tiếng Trung Quốc không giống nhau (Q5, Mean = 2.75), có thái độ trung dung trước quan niệm học tiếng Trung Quốc nhất định phải học tại Trung Quốc (Q11, Mean = 2.99). Đại đa số sinh viên cho rằng học tiếng Trung Quốc phải tìm hiểu văn hoá Trung Quốc $(\mathrm{Q} 8$, Mean $=4.26)$. Điều này cho thấy sinh viên đã nhận thức được mối quan hệ mật thiết giữa ngôn ngữ và văn hoá. Vì vậy, giảng viên cần giới thiệu văn hoá Trung Quốc, gắn các yếu tố văn hoá vào quá trình giảng dạy tiếng Trung Quốc, giải thích mối liên hệ giữa chữ và nghĩa của một số chữ Hán tiêu biểu trong thời lượng cho phép để giảm thiểu áp lực chữ Hán khó học, tăng cường và duy trì hứng thú học tập tiếng Trung Quốc của sinh viên.

Về trọng tâm trong học tập tiếng Trung Quốc, sinh viên rất xem trọng việc học từ vựng $(\mathrm{Q} 16$, Mean = 4.56), không xem trọng việc học ngữ pháp $(\mathrm{Q} 20$, Mean = 2.71) hay dịch Việt - Trung
$(\mathrm{Q} 26$, Mean $=2.53)$. Điều này có thể dễ hiểu vì như trên đã nói tiếng Việt và tiếng Trung Quốc đều là ngôn ngữ thuộc loại hình đơn lập, đặc điểm ngữ pháp của hai ngôn ngữ này có khá nhiều điểm tương đồng, sinh viên không phải mất quá nhiều thời gian để ghi nhớ các quy tắc ngữ pháp. Ngoài ra, tiếng Trung Quốc chỉ là ngoại ngữ thứ hai của sinh viên, chuẩn đầu ra chương trình đào tạo ngành Ngôn ngữ Anh của Trường Đại học Ngân hàng TP. Hồ Chí Minh không yêu cầu sinh viên phải có kĩ năng dịch Việt - Trung. Ngược lại, từ vựng là vật liệu xây dựng nên ngôn ngữ và lời nói, sinh viên cần tích luỹ một lượng lớn từ vựng tiếng Trung Quốc mới có thể nâng cao năng lực tiếng Trung Quốc của mình.

\subsubsection{Chiến lự̛c học tập-giao tiếp}

Trong BALLI có 8 câu hỏi về phương diện chiến lược học tập - giao tiếp. Tỉ lệ phần trăm của các lựa chọn, Mean và SD của 8 câu hỏi này như sau (xem bảng 4$)$ :

Bảng 4. Kết quả điều tra về chiến lược học tập - giao tiếp

\begin{tabular}{ccccccccc}
\hline \multirow{2}{*}{ Mã câu hỏi } & \multicolumn{9}{c}{ Tỉ lệ phần trăm } & \multirow{2}{*}{ Mean } & SD \\
\cline { 2 - 5 } & 1 & 2 & 3 & 4 & 5 & & \\
\hline Q7 & 0 & 0.6 & 3.4 & 23.7 & 72.3 & 4.68 & 0.567 \\
Q9 & 9.0 & 13.0 & 36.7 & 20.3 & 21.0 & 3.31 & 1.201 \\
Q12 & 1.1 & 7.9 & 32.8 & 47.5 & 10.7 & 3.59 & 0.829 \\
Q13 & 1.1 & 9.6 & 13.6 & 53.7 & 22.0 & 3.86 & 0.909 \\
Q17 & 1.1 & 0 & 0 & 7.3 & 91.5 & 4.88 & 0.491 \\
Q18 & 11.9 & 28.2 & 22.6 & 31.1 & 6.2 & 2.92 & 1.147 \\
Q19 & 18.1 & 18.6 & 16.4 & 19.2 & 27.7 & 3.20 & 1.477 \\
Q21 & 1.7 & 6.2 & 40.7 & 29.4 & 22.0 & 3.64 & 0.950 \\
\hline
\end{tabular}


Bảng 4 cho thấy sinh viên đánh giá rất cao tầm quan trọng của ngữ âm $(\mathrm{Q} 7$, Mean $=4.68)$. Ngữ âm có vai trò vô cùng quan trọng trong học tập ngôn ngữ, phát âm không chuẩn rất có thể sẽ ảnh hưởng đến sự biểu đạt của lời nói, đồng thời cũng ảnh hưởng đến việc nghe hiểu, ngược lại nếu phát âm chính xác sẽ rất dễ lưu lại ấn tượng tốt cho người nghe. Việc chú trọng tính chính xác trong phát âm sẽ rất hữu ích cho việc học tiếng Trung Quốc của sinh viên, song nếu quá chú trọng tính chính xác trong phát âm sẽ ảnh hưởng đến tính lưu loát trong giao tiếp bằng tiếng Trung Quốc của sinh viên (Gu Ju-hua (顾菊华), 2007; Ding An-qi (丁安 琪) và Wu Si-na (吴思娜), 2011). Bảng 4 còn cho thấy, sinh viên cũng rất chú trọng việc lặp lại và luyện tập nhiều lần $(\mathrm{Q} 17$, Mean $=4.88)$. Đa số sinh viên khi nghe người khác nói tiếng Trung Quốc, sẽ nói cùng với họ (Q12, Mean = 3.59 ) và thích sử dụng chiến lược đoán từ khi gặp từ mới (Q13, Mean = 3.86). Một bộ phận sinh viên không đồng ý quan niệm "Tôi rất ngại dùng tiếng Trung Quốc để nói chuyện với người khác" (Q18, Mean = 2.92). Kết quả này giống với kết quả nghiên cứu của Ding An-qi (丁安琪) (2010), Lin Lun-lun (林伦伦) và Ren Meng-ya (任梦雅) (2010), Ding An-qi (丁安琪) và Wu Si-na (吴思娜) (2011).

Đa số sinh viên cho rằng không nên sử dụng tiếng Trung Quốc nếu không thể biểu đạt chính xác bằng tiếng Trung Quốc $(\mathrm{Q} 9$, Mean $=3.31$ ), vì chuyên ngành của sinh viên là Ngôn ngữ Anh, đây là ngôn ngữ phổ biến nhất thế giới. Đa số sinh viên tán thành quan niệm "Nếu cho phép người mới học mắc lỗi, thì sau này họ khó có thể nói chính xác" (Q19, Mean $=3.20$ ). Kết quả này không giống kết quả nghiên cứu của Ding An-qi (丁安琪) (2010), Ding An-qi (丁安琪) và Wu Si-na (吴思娜) (2011). Qua đó cho thấy không giống với sinh viên Hàn Quốc và sinh viên Malaysia, sinh viên Trường Đại học Ngân hàng TP. Hồ Chí Minh yêu cầu đối với bản thân tương đối cao, không cho phép mắc sai lầm trong sử dụng tiếng Trung Quốc. Điều này không có lợi cho việc bồi dưỡng năng lực giao tiếp ngôn ngữ, nhưng lại hữu ích cho việc nâng cao tính chính xác trong biểu đạt ngôn ngữ, từ đó nâng cao trình độ tiếng Trung Quốc của sinh viên.

\subsection{5. Động co học tập}

Trong BALLI có 4 câu hỏi về phương diện động cơ học tập. Tỉ lệ phần trăm của các lựa chọn, Mean và $\mathrm{SD}$ của 4 câu hỏi này như sau (xem bảng 5):

Bảng 5. Kết quả điều tra về động cơ học tập

\begin{tabular}{cccccccc}
\hline \multirow{2}{*}{ Mã câu hỏi } & \multicolumn{9}{c}{ Tỉ lệ phần trăm } & \multirow{2}{*}{ Mean } & \multirow{2}{*}{ SD } \\
\cline { 2 - 5 } & 1 & 2 & 3 & 4 & 5 & & \\
\hline Q23 & 0 & 1.7 & 4.0 & 24.2 & 70.1 & 4.63 & 0.646 \\
Q27 & 0 & 0.6 & 3.4 & 30.5 & 65.5 & 4.61 & 0.584 \\
Q30 & 5.6 & 19.8 & 46.9 & 24.3 & 3.4 & 3.00 & 0.898 \\
Q31 & 1.1 & 6.2 & 10.7 & 46.9 & 35.0 & 4.08 & 0.897 \\
\hline
\end{tabular}

Bảng 5 cho thấy đại đa số sinh viên cho rằng nếu học tốt tiếng Trung Quốc, sẽ có nhiều cơ hội sử dụng tiếng Trung Quốc (Q23, Mean = $4.63)$, có thể tìm được công việc tốt hơn (Q27, Mean $=4.61)$, và có thể hiểu hơn về người Trung Quốc (Q31, Mean = 4.08). Qua đó cho thấy sinh viên có động cơ học tập tiếng Trung Quốc rất cao. Điều này rất có ích cho việc học tiếng Trung Quốc.

Song, sinh viên có đánh giá ở mức độ trung bình (Mean = 3.00) trước câu hỏi "Người Việt Nam cho rằng, biết nói tiếng Trung Quốc rất 
quan trọng" (Q30). Đây có thể là vì khách thể tham gia điều tra là sinh viên ngành Ngôn ngữ Anh, họ cho rằng tiếng Anh là quan trọng nhất, tầm quan trọng của tiếng Trung Quốc không bằng tiếng Anh.

\section{2. Ảnh hưởng của các nhân tố cá thể đối với quan niệm học tập}

\subsubsection{Anh hưởng của giơoi tính đối với quan niệm học tập}

Sau khi tiến hành kiểm định giả thuyết về trị trung bình của hai tổng thể - trường hợp mẫu độc lập (Independent - samples T-test), chúng tôi phát hiện giữa sinh viên nam và sinh viên nữ có sự khác biệt có ý nghĩa $(\mathrm{p}<0.05)$ ở các nội dung sau (xem bảng 6):

Bảng 6. Những nội dung khác biệt có ý nghĩa về giới tính

\begin{tabular}{ccccc}
\hline Mã câu hỏi & Giới tính & Mean & $\mathrm{t}$ & $\mathrm{p}$ \\
\hline \multirow{2}{*}{ Q7 } & Nam & 4.24 & -2.523 & 0.020 \\
& Nũ̃ & 4.74 & & \\
Q23 & Nam & 4.33 & -2.246 & 0.026 \\
\hline
\end{tabular}

Bảng 6 cho thấy sinh viên nam và sinh viên nữ đều rất chú trọng vào tính chính xác trong phát âm $(\mathrm{Q} 7)$ và rất có niềm tin vào cơ hội sử dụng tiếng Trung Quốc (Q23), song mức độ đồng ý của sinh viên nữ ở hai nội dung này đều nổi trội hơn sinh viên nam.

\subsubsection{Anh hưởng của thời gian học đối với quan niệm học tộp}

Bảng 7. Những nội dung khác biệt có ý nghĩa về thời gian học

\begin{tabular}{|c|c|c|c|c|}
\hline Mã câu hỏi & Thời gian hoc & Mean & $\mathrm{t}$ & $\mathrm{p}$ \\
\hline \multirow{2}{*}{ Q7 } & Năm thứ hai & 4.84 & \multirow{2}{*}{3.764} & \multirow{2}{*}{0.000} \\
\hline & Năm thứ ba & 4.53 & & \\
\hline \multirow{2}{*}{ Q11 } & Năm thứ hai & 2.78 & \multirow{2}{*}{-2.089} & \multirow{2}{*}{0.038} \\
\hline & Năm thứ ba & 3.20 & & \\
\hline \multirow{2}{*}{ Q12 } & Năm thứ hai & 3.77 & \multirow{2}{*}{2.864} & \multirow{2}{*}{0.005} \\
\hline & Năm thứ ba & 3.42 & & \\
\hline \multirow{2}{*}{ Q13 } & Năm thứ hai & 4.01 & \multirow{2}{*}{2.205} & \multirow{2}{*}{0.029} \\
\hline & Năm thứ ba & 3.71 & & \\
\hline \multirow{2}{*}{ Q20 } & Năm thứ hai & 2.26 & \multirow{2}{*}{-5.656} & \multirow{2}{*}{0.000} \\
\hline & Năm thứ ba & 3.14 & & \\
\hline \multirow{2}{*}{ Q22 } & Năm thứ hai & 1.83 & \multirow{2}{*}{-4.140} & \multirow{2}{*}{0.000} \\
\hline & Năm thứ ba & 2.47 & & \\
\hline \multirow[b]{2}{*}{ Q24 } & Năm thứ hai & 3.15 & \multirow{2}{*}{-3.150} & \multirow{2}{*}{0.002} \\
\hline & Năm thứ ba & 3.68 & & \\
\hline \multirow{2}{*}{ Q29 } & Năm thứ hai & 1.84 & \multirow{2}{*}{-2.601} & \multirow{2}{*}{0.010} \\
\hline & Năm thứ ba & 2.25 & & \\
\hline \multirow{2}{*}{ Q32 } & Năm thứ hai & 3.41 & \multirow{2}{*}{-2.950} & \multirow{2}{*}{0.004} \\
\hline & Năm thứ ba & 3.85 & & \\
\hline \multirow{2}{*}{ Q34 } & Năm thứ hai & 4.36 & \multirow{2}{*}{2.110} & \multirow{2}{*}{0.036} \\
\hline & Năm thứ ba & 4.05 & & \\
\hline
\end{tabular}


Bảng 7 cho thấy sinh viên năm thứ hai có điểm trung bình ở các nội dung "Khi học tiếng Trung Quốc, ngữ âm chính xác rất quan trọng" (Q7), "Nếu tôi nghe thấy có người nói tiếng Trung Quốc, tôi sẽ nói cùng với họ" (Q12), "Nếu có một từ tiếng Trung Quốc tôi không biết, tôi sẽ đoán nghĩa của nó dựa vào quan hệ giữa chữ và nghĩa của từ đó" (Q13), "Ai cũng có thể học tốt một ngoại ngữ" (Q34), nổi trội hơn sinh viên năm thứ ba, ngược lại sinh viên năm thứ ba có điểm trung bình ở các nội dung "Tốt nhất là học tiếng Trung Quốc tại Trung Quốc" (Q11), "Học tiếng Trung Quốc là học rất nhiều ngữ pháp" (Q20), "Nữ giới học ngoại ngữ giỏi hơn nam giới” (Q22), "Nói một ngoại ngữ dễ hơn nghe, đọc, viết ngoại ngữ đó" (Q24), "Người có sở trường về toán và khoa học tự nhiên, không có sở trường trong việc học ngoại ngữ" (Q29), "Người biết nói hơn một ngoại ngữ rất thông minh" (Q32) nổi trội hơn sinh viên năm thứ hai.

Kết quả trên cho thấy sinh viên năm thứ hai và sinh viên năm thứ ba đều cho rằng ai cũng có thể học tốt ngoại ngữ, nhưng so với

sinh viên năm thứ hai, sinh viên năm thứ ba tin rằng có một bộ phận người có năng lực học tập ngoại ngữ tốt hơn. Sinh viên năm thứ hai chú trọng về tính chính xác của ngữ âm cao hơn sinh viên năm thứ ba, còn sinh viên năm thứ ba chú trọng về việc học ngữ pháp hơn sinh viên năm thứ hai. Sinh viên năm thứ ba đánh giá cao môi trường ngôn ngữ đích hơn sinh viên năm thứ hai. Sinh viên năm thứ hai thích sử dụng chiến lược đoán nghĩa của từ và thích giao tiếp với người nói tiếng Trung Quốc hơn sinh viên năm thứ ba. Có thể thấy thời gian học tiếng Trung Quốc đã có tác động nhất định đến quan niệm học tập ngoại ngữ thứ hai - tiếng Trung Quốc của sinh viên.

\subsubsection{Anh hưởng của vùng miền đối với quan niệm học tập}

Sau khi tiến hành phân tích phương sai một yếu tố (oneway ANOVA), chúng tôi phát hiện giữa sinh viên các vùng miền có sự khác biệt có ý nghĩa $(p<0.05)$ ở các nội dung sau (xem bảng 8 ):

Bảng 8 . Những nội dung khác biệt có ý nghĩa về vùng miền

\begin{tabular}{ccccc}
\hline Mã câu hỏi & Vùng miền & Mean & $\mathrm{F}$ & $\mathrm{p}$ \\
\hline \multirow{2}{*}{ Q11 } & Miền bắc & 3.12 & \multirow{2}{*}{0.672} & 0.027 \\
& Miền trung & 2.82 & & \\
& Miền nam & 3.79 & & \multirow{2}{*}{0.014} \\
& Miền bắc & 2.88 & 4.339 & \\
\multirow{2}{*}{ Q15 } & Miền trung & 2.47 & & 0.007 \\
& Miền nam & 2.93 & \multirow{2}{*}{5.063} & \\
\hline
\end{tabular}

Bảng 8 cho thấy ở nội dung "Tốt nhất là học tiếng Trung Quốc tại Trung Quốc" (Q11), sinh viên đến từ các tỉnh, thành miền bắc và miền nam đều tán thành quan niệm này, nhưng sinh viên đến từ các tỉnh, thành miền trung thì ngược lại, họ không cho rằng nhất định phải đến Trung Quốc học tiếng Trung Quốc.
Bảng 8 còn cho thấy sinh viên cả ba miền đều không đồng ý quan niệm "Tôi có khả năng đặc biệt trong việc học ngoại ngữ” (Q15) và "Nữ giới học ngoại ngữ giỏi hơn nam giới" (Q22). Trong đó, sinh viên đến từ các tỉnh, thành miền trung có mức độ đồng ý thấp nhất. Họ không cho rằng mình có năng lực học tập 
ngoại ngữ đặc biệt, cũng không cho rằng nữ giới học ngoại ngữ giỏi hơn nam giới.

\subsection{Mối quan hệ giữa kết quả học tập với quan niệm học tập}

Chúng tôi tiến hành phân tích tương quan Pearson giữa kết quả học tập và quan niệm học tập của sinh viên. Kết quả cho thấy tồn tại mối tương quan giữa kết quả học tập và các nội dung sau (xem bảng 9):

Bảng 9. Phân tích mối tương quan giữa kết quả học tập và quan niệm học tập

\begin{tabular}{llll}
\hline & Q4 & Q6 & Q18 \\
\hline Pearson Correlation & 0.201 & 0.355 & -0.222 \\
Sig. (2-tailed) & 0.007 & 0.000 & 0.003 \\
\hline
\end{tabular}

Bảng 9 cho thấy tồn tại mối tương quan thuận giữa kết quả học tập và các nội dung "Tiếng Trung Quốc là ngoại ngũ̃ dễ học" (Q4), "Tôi tin tôi có thể học tốt tiếng Trung Quốc" (Q6), và tồn tại mối tương quan nghịch giữa kết quả học tập và quan niệm "Tôi rất ngại dùng tiếng Trung Quốc để nói chuyện với người khác" (Q18). Qua đó cho thấy nếu sinh viên cho rằng tiếng Trung Quốc là ngoại ngữ dễ học, có niềm tin bản thân trong quá trình học tập và chủ động sử dụng tiếng Trung Quốc trong giao tiếp, thì sẽ có kết quả học tập tốt hơn. Ngược lại, nếu sinh viên tự kỉ ám thị tiếng Trung Quốc rất khó và không dám sử dụng tiếng Trung Quốc trong giao tiếp sẽ không có được kết quả học tập tốt.

\section{Kết luận}

Từ những phân tích trên, chúng ta có thể thấy quan niệm học tập ngoại ngữ thứ hai tiếng Trung Quốc của sinh viên ngành Ngôn ngữ Anh, Trường Đại học Ngân hàng TP. Hồ Chí Minh như sau: thứ nhất, có ngoại ngữ dễ học, có ngoại ngữ khó học, tiếng Trung Quốc là ngoại ngữ tương đối dễ học đối với người Việt Nam; thứ hai, mỗi người có năng lực học tập ngoại ngữ khác nhau, trẻ em có năng lực học tập ngoại ngữ tốt hơn người lớn, tuy không cho rằng bản thân có năng lực đặc biệt trong học tập ngoại ngữ, nhưng tin rằng mình có thể học tốt tiếng Trung Quốc; thứ ba, chú trọng tính chính xác của ngữ âm, chú trọng việc học từ vựng và văn hoá Trung Quốc, không chú trọng ngữ pháp tiếng Trung Quốc; thứ tư, động cơ học tập rất cao, cho rằng học tiếng Trung Quốc có ích cho bản thân. Về phương diện giới tính, sinh viên nữ chú trọng đến tính chính xác về mặt ngữ âm và có niềm tin vào cơ hội sử dụng tiếng Trung Quốc hơn sinh viên nam. Về phương diện thời gian học, sinh viên năm thứ hai chú trọng về tính chính xác của ngữ âm, thích sử dụng chiến lược đoán nghĩa của từ và thích giao tiếp với người nói tiếng Trung Quốc cao hơn sinh viên năm thứ ba; ngược lại sinh viên năm thứ ba chú trọng về việc học ngữ pháp hơn sinh viên năm thứ hai. Về phương diện vùng miền, sinh viên đến từ các tỉnh, thành miền bắc và miền nam cho rằng nên đến Trung Quốc học tiếng Trung Quốc, sinh viên đến từ các tỉnh, thành miền trung thì có quan niệm ngược lại. Các quan niệm tiếng Trung Quốc dễ học, tin mình có thể học tốt tiếng Trung Quốc và chủ động sử dụng tiếng Trung Quốc trong giao tiếp có ảnh hưởng tích cực đến kết quả học tập của sinh viên.

\section{Kiến nghị}

Trên cơ sở các kết quả nghiên cứu trên đây, chúng tôi xin đưa ra một số kiến nghị sau:

Thứ nhất, giảng viên cần cho sinh viên biết quan niệm "Trẻ em học ngoại ngữ dễ hơn người lớn” vẫn còn là một vấn đề gây tranh cãi. Marinova - Todd, Marshall và Snow 
(2000) sau khi phân tích các kết quả nghiên cứu về ảnh hưởng của tuổi tác đối với việc học ngoại ngũ đã chỉ ra rằng, sự khác biệt về tuổi tác phản ánh sự khác biệt trong tình hình học tập hơn là năng lực học tập, thực tế cho thấy trẻ em học ngôn ngữ mới với tốc độ chậm và ít nỗ lực hơn người lớn, người lớn thất bại trong việc học ngôn ngữ mới là vì họ không có động cơ học tập cao, không dành nhiều thời gian và sức lực cho việc học.

Thứ hai, bên cạnh việc chú trọng tính chính xác về mặt phát âm, giảng viên cũng cần chú trọng hơn về giảng dạy ngữ pháp. Ngữ pháp tiếng Việt và tiếng Trung Quốc tuy có nhiều điểm tương đồng, song cũng có những điểm dị biệt. Nếu sinh viên nắm vững các quy tắc ngữ pháp tiếng Trung Quốc, hiểu được những khác biệt của hai ngôn ngữ Việt - Trung sẽ rất hữu ích cho việc nâng cao năng lực tiếng Trung Quốc của sinh viên.

Thứ ba, giảng viên cần xây dựng cho sinh viên niềm tin "tôi có thể học tốt tiếng Trung Quốc" và không ngừng kích thích tính chủ động sử dụng tiếng Trung Quốc trong giao tiếp của sinh viên. Kết quả phân tích mối tương quan giữa kết quả học tập và quan niệm học tập (xem mục 4.3) cho thấy, những sinh viên có kết quả học tập tốt là những sinh viên có quan niệm "Tiếng Trung Quốc dễ học", đồng thời tự tin và chủ động sử dụng tiếng Trung Quốc.

\section{Tài liệu tham khảo}

\section{Tiếng Việt}

Bùi Mạnh Hùng (2008). Ngôn ngũ học đối chiếu. Hà Nội: Nhà xuất bản Giáo dục.

\section{Tiếng Anh}

Amuzie, G. L. \& P. Winke (2009). Changes in language learning beliefs as a result of study abroad. System, 37(3), 366-379.

Bacon, S. M. \& Finnemann, M. D. (1990). A study of attitudes, motives and strategies of university foreign language students and their disposition to authentic oral and written input. Modern Language Journal, 74(4), 459-473.

Bernat, E. (2006). Assessing EAP learners' beliefs about language learning in the Australian context. Asian EFL Journal, 8(2), 202-227.

Bernat, E. \& Lloyd, R. (2007). Exploring the gender effect on EFL learners' beliefs about language learning. Australian Journal of Educational \& Developmental Psychology, 7, 79-91.

Horwitz, E. K. (1985). Using student beliefs about language learning and teaching in the foreign language methods course. Foreign Language Annals, 18(4), 333-340.

Jee, M. J. (2014). Affective factors in Korean as a foreign language: Anxiety and beliefs. Language, Culture and Curriculum, 27(2), 182-195.

Marinova - Todd, S. H., Marshall, D. B. \& Snow, C. E. (2000). Three Misconceptions about Age and L2 Learning. TESOL Quarterly, 34(1), 9-34.

Mori, Y. (1999). Epistemological beliefs and language learning beliefs: What do language learners believe about their learning? Language Learning, 49(3), 377-415.

Peacock, M. (2001). Pre-service ESL teachers' beliefs about second language learning: A longitudinal study. System, 29(2), 177-195.

Tanaka, K. \& Ellis, R. (2003). Study-abroad language proficiency and learner beliefs about language learning. JALT Journal, 25(1), 63-85.

Victori, M. \& Lockheart W. (1995). Enhancing metacognition in self - Directed language learning. System, 23(2), 223-234.

Wenden, A. (1987). How to be a successful language learner Insights and Prescriptions from L2 learners. In Wenden, A. \& Rubin, J. (Ed.), Learning Strategies in Language Learning (pp. 103-117). New Jersey: Prentice Hall.

Wenden, A. (1991). Learner Strategies for Learner Autonomy. New Jersey: Prentice Hall.

\section{Tiếng Trung Quốc}

曹贤文 \& 吴淮南 (2002). 留学生的几项个体差异变 量与学习成就的相关分析. 暨南大学华文学院 学报, (3), 11-16.

丁安琪 \& 吴思娜 (2011). 汉语作为第二语言学习者 实证研究. 北京: 世界图书出版公司.

丁安琪. (2010). 汉语作为第二语言学习者研究. 北京: 
世界图书出版公司.

高彦德, 李国强 \& 郭旭 (1993). 外国人学习与使用

汉语情况调查研究报告. 北京: 北京语言学院出 版社.

顾菊华. (2007). 英语学习行为研究. 昆明: 云南大学 出版社.

林伦伦 \& 任梦雅 (2010). 非洲留学生汉语学习观念

的社会语言学调查. 韩山师范学院学报, 3l(5),
32-37.

三井明子 \& 邵明明 (2019). 日本华裔和非华裔的汉 语学习观念对比研究. 国际汉语教育(中英文), 4(03), 51-61.

吴艳 \& 孙莉明 (2010). 韩国留学生汉语学习观念 的调查分析. 沈阳农业大学学报 (社会科学版), 12(2), 196-198.

张慧 (2011). 目的语环境下中亚留学生汉语学习观

念的调查和分析. 读与写杂志, 8(10), 39-40.

\title{
A STUDY ON THE LEARNING BELIEFS OF CHINESE AS A SECOND FOREIGN LANGUAGE FOR ENGLISH MAJORED STUDENTS OF BANKING UNIVERSITY HO CHI MINH CITY
}

\author{
Luu Hon $\mathrm{Vu}$ \\ Faculty of Foreign Languages, Banking University HCMC \\ 36 Ton That Dam, District 1, Ho Chi Minh city, Vietnam
}

\begin{abstract}
The research surveyed the learning beliefs of Chinese as a second foreign language for English majored students of Banking University Ho Chi Minh city. Based on the theory of beliefs in foreign language learning by Horwitz (1985), we conducted a questionnaire survey with 177 students. The questionnaire results indicate that: firstly, Chinese is relatively easy to learn; secondly, children have better language learning capacity than adults; thirdly, focus on phonetics, vocabulary and culture, not grammar; fourthly, learning Chinese is useful for themselves. Female students focus on phonetics more than male students. Second-year students focus on phonetics more than third-year students, but not more grammar like third-year students. Unlike students from the central region, students from the northern and southern regions said that they must come to China to learn Chinese. The belief that "Chinese language is easy to learn", the confident and proactive attitude of using Chinese language has a positive impact on students' learning results.

Keywords: learning beliefs; Chinese; second foreign language; Vietnamese students
\end{abstract}




\section{PHỤ LỤC}

\section{BẢNG ĐIỀU TRA QUAN NIỆM HỌC TẬP NGOẠI NGỬ THƯ' HAI - TIÊNG TRUNG QUỐC}

Căn cứ vào tình hình thực tế, bạn hãy khoanh tròn chữ số biểu thị mức độ đồng ý cho các câu bên dưới.
$1==$
$===2==$
$===3==$
$===4==$
$==5$

Hoàn toàn không Không đồng ý Bình thường

Đồng ý

Hoàn toàn đồng ý

đồng ý

\begin{tabular}{|c|c|c|c|c|c|c|}
\hline Q1 & Trẻ em học ngoại ngữ dễ hơn người lớn. & 1 & 2 & 3 & 4 & 5 \\
\hline Q2 & Có một số người có khả năng bẩm sinh đặc biệt trong học ngoại ngữ. & 1 & 2 & 3 & 4 & 5 \\
\hline Q3 & Có một số ngoại ngữ dễ học, có một số ngoại ngữ khó học. & 1 & 2 & 3 & 4 & 5 \\
\hline Q4 & Tiếng Trung Quốc là ngoại ngữ dễ học. & 1 & 2 & 3 & 4 & 5 \\
\hline Q5 & Tiếng Trung Quốc và tiếng Việt không giống nhau. & 1 & 2 & 3 & 4 & 5 \\
\hline Q6 & Tôi tin tôi có thể học tốt tiếng Trung Quốc. & 1 & 2 & 3 & 4 & 5 \\
\hline Q7 & Khi học tiếng Trung Quốc, ngữ âm chính xác rất quan trọng. & 1 & 2 & 3 & 4 & 5 \\
\hline Q8 & Học tiếng Trung Quốc phải tìm hiểu văn hoá Trung Quốc. & 1 & 2 & 3 & 4 & 5 \\
\hline Q9 & $\begin{array}{l}\text { Những điều không thể biểu đạt chính xác bằng tiếng Trung Quốc thì } \\
\text { đừng nói bằng tiếng Trung Quốc. }\end{array}$ & 1 & 2 & 3 & 4 & 5 \\
\hline Q10 & Người biết một ngoại ngữ sẽ dễ học một ngoại ngữ khác hơn. & 1 & 2 & 3 & 4 & 5 \\
\hline Q11 & Tốt nhất là học tiếng Trung Quốc tại Trung Quốc. & 1 & 2 & 3 & 4 & 5 \\
\hline Q12 & Nếu tôi nghe thấy có người nói tiếng Trung Quốc, tôi sẽ nói cùng với họ. & 1 & 2 & 3 & 4 & 5 \\
\hline Q13 & $\begin{array}{l}\text { Nếu có một từ tiếng Trung Quốc tôi không biết, tôi sẽ đoán nghĩa } \\
\text { của nó dựa vào quan hệ giữa chữ và nghĩa của từ đó. }\end{array}$ & 1 & 2 & 3 & 4 & 5 \\
\hline Q14 & $\begin{array}{l}\text { Nếu một người dùng một tiếng mỗi ngày để học tiếng Trung Quốc, } \\
\text { theo bạn bao lâu người đó sẽ sử dụng thành thạo tiếng Trung Quốc? } \\
(1)<1 \text { năm; (2) 1-2 năm; (3) 3-5 năm; (4) 5-10 năm; (5) Không thể. }\end{array}$ & 1 & 2 & 3 & 4 & 5 \\
\hline Q15 & Tôi có khả năng đặc biệt trong việc học ngoại ngũ. & 1 & 2 & 3 & 4 & 5 \\
\hline Q16 & Điều quan trọng nhất trong học tiếng Trung Quốc là học từ vựng. & 1 & 2 & 3 & 4 & 5 \\
\hline Q17 & Lặp lại và luyện tập nhiều lần rất quan trọng. & 1 & 2 & 3 & 4 & 5 \\
\hline Q18 & Tôi rất ngại dùng tiếng Trung Quốc để nói chuyện với người khác. & 1 & 2 & 3 & 4 & 5 \\
\hline Q19 & $\begin{array}{l}\text { Nếu cho phép người mới học mắc lỗi, thì sau này họ khó có thể nói } \\
\text { chính xác. }\end{array}$ & 1 & 2 & 3 & 4 & 5 \\
\hline Q20 & Học tiếng Trung Quốc là học rất nhiều ngữ pháp. & 1 & 2 & 3 & 4 & 5 \\
\hline Q21 & $\begin{array}{l}\text { Luyện tập tiếng Trung Quốc trong phòng thực nghiệm ngữ âm rất } \\
\text { quan trọng. }\end{array}$ & 1 & 2 & 3 & 4 & 5 \\
\hline Q22 & Nữ giới học ngoại ngữ giỏi hơn nam giới. & 1 & 2 & 3 & 4 & 5 \\
\hline Q23 & $\begin{array}{l}\text { Nếu nói tốt tiếng Trung Quốc, tôi sẽ có nhiều cơ hội sử dụng tiếng } \\
\text { Trung Quốc. }\end{array}$ & 1 & 2 & 3 & 4 & 5 \\
\hline Q24 & Nói một ngoại ngữ dễ hơn nghe, đọc, viết ngoại ngữ đó. & 1 & 2 & 3 & 4 & 5 \\
\hline
\end{tabular}




\begin{tabular}{|l|l|l|l|l|l|l|}
\hline Q25 & Học tiếng Trung Quốc không giống học các ngoại ngữ khác. & 1 & 2 & 3 & 4 & 5 \\
\hline Q26 & $\begin{array}{l}\text { Điều quan trọng nhất trong học tiếng Trung Quốc là học cách làm } \\
\text { thế nào để dịch tiếng Việt sang tiếng Trung Quốc. }\end{array}$ & 1 & 2 & 3 & 4 & 5 \\
\hline Q27 & Nếu nói tốt tiếng Trung Quốc, tôi có thể tìm được công việc tốt hơn. & 1 & 2 & 3 & 4 & 5 \\
\hline Q28 & Đọc và viết tiếng Trung Quốc dễ hơn nghe và nói tiếng Trung Quốc. & 1 & 2 & 3 & 4 & 5 \\
\hline Q29 & $\begin{array}{l}\text { Người có sở trường về toán và khoa học tự nhiên, không có sở } \\
\text { trường trong việc học ngoại ngữ. }\end{array}$ & 1 & 2 & 3 & 4 & 5 \\
\hline Q30 & Người Việt Nam cho rằng, biết nói tiếng Trung Quốc rất quan trọng. & 1 & 2 & 3 & 4 & 5 \\
\hline Q31 & Học tiếng Trung Quốc có thể hiểu hơn về người Trung Quốc. & 1 & 2 & 3 & 4 & 5 \\
\hline Q32 & Người biết nói hơn một ngoại ngữ rất thông minh. & 1 & 2 & 3 & 4 & 5 \\
\hline Q33 & Người Việt Nam có sở trường học ngoại ngữ. & 1 & 2 & 3 & 4 & 5 \\
\hline Q34 & Ai cũng có thể học tốt một ngoại ngữ. & 1 & 2 & 3 & 4 & 5 \\
\hline
\end{tabular}

